Running Head: Maximal inverse subsemigroups

\title{
Maximal inverse subsemigroups of the symmetric inverse semigroup on a finite-dimensional vector space \\ Suzana Mendes-Gonçalves ${ }^{* \dagger}$
}

Centro de Matemática, Universidade do Minho, 4710 Braga, Portugal

and

\section{R P Sullivan}

School of Mathematics \& Statistics, University of Western Australia

Nedlands 6009, Australia

\begin{abstract}
Yang (1999) classified the maximal inverse subsemigroups of all the ideals of the symmetric inverse semigroup $I(X)$ defined on a finite set $X$. Here we do the same for the semigroup $I(V)$ of all one-to-one partial linear transformations of a finitedimensional vector space. We also show that $I(X)$ is almost never isomorphic to $I(V)$ for any set $X$ and any vector space $V$, and prove that any inverse semigroup can be embedded in some $I(V)$.
\end{abstract}

AMS Primary Classification: 20M20; Secondary: 15A04.

Keywords: maximal inverse subsemigroups, linear transformation semigroups

† Correspondence: Suzana Mendes-Gonçalves, Departamento de Matematica, Campus de Gualtar - Universidade do Minho, 4710-057 Braga, Portugal; E-mail: smendes@math.uminho.pt.

* This paper forms part of work by the first author for a PhD supervised by the second author; the first author acknowledges the support of the Portuguese Foundation for Science and Technology (FCT) through the research program POCTI. 


\section{Introduction}

Suppose $V$ is a vector space and let $P(V)$ denote the set of all partial linear transformations of $V$ : that is, all linear transformations $\alpha$ whose domain, dom $\alpha$, and range, ran $\alpha$, are subspaces of $V$. As for partial transformations of a set (compare [2] Vol. 1, p. 29) we define the composition $\alpha \circ \beta$ of $\alpha, \beta \in P(V)$ to be the linear transformation with domain $U=(\operatorname{ran} \alpha \cap \operatorname{dom} \beta) \alpha^{-1}$ such that, for all $u \in U$,

$$
u(\alpha \circ \beta)=(u \alpha) \beta,
$$

and we often write $\alpha \circ \beta$ more simply as $\alpha \beta$. Clearly, $U$ is a subspace of $V$ and $\alpha \circ \beta \in P(V)$ if $\alpha, \beta \in P(V)$. Also, $(\alpha \circ \beta) \circ \gamma=\alpha \circ(\beta \circ \gamma)$ for all $\alpha, \beta, \gamma \in P(V)$, so $(P(V), \circ)$ is a semigroup (unless stated otherwise, we use the notation and terminology of [2]). As usual, we let $\operatorname{ker} \alpha=\{u \in \operatorname{dom} \alpha: u \alpha=0\}$ and $\operatorname{rank} \alpha=\operatorname{dim} \operatorname{ran} \alpha$.

There is a natural subsemigroup of $P(V)$ - namely, $I(V)$ - which consists of all one-toone partial linear transformations of $V$ (that is, all $\alpha \in P(V)$ such that ker $\alpha=\{0\}$ ); and it is easy to see that $I(V)$ is an inverse semigroup (that is, for each $\alpha \in I(V)$ there exists a unique $\beta \in I(V)$, namely $\beta=\alpha^{-1}$, such that $\alpha=\alpha \beta \alpha$ and $\beta=\beta \alpha \beta$ ). Some properties of $I(V)$ were studied in [8] in the context of 'independence algebras'. In fact, $I(V)$ can be regarded as a linear version of the well-known 'symmetric inverse semigroup' $I(X)$ defined on a set $X$, and we adopt this approach here. Note that we use the ' $V$ ' in $I(V)$ to denote the fact that we are considering linear transformations.

For example, it is well-known that the ideals of $I(X)$ are the sets

$$
I_{r}(X)=\{\alpha \in I(X): \operatorname{rank} \alpha \leq r\}
$$

where $0 \leq r \leq|X|$ and, for $\alpha \in I(X)$, rank $\alpha=|\operatorname{ran} \alpha|$. Likewise, following [2] Vol. 1 , p. 57 , Exercise 6 , it can be shown that, if $\operatorname{dim} V=n<\aleph_{0}$, then the ideals of $I(V)$ are precisely the sets

$$
I_{r}(V)=\{\alpha \in I(V): \operatorname{rank} \alpha \leq r\}
$$

where $0 \leq r \leq n$ (in a small way, this extends a remark in [8] p. 427). Note that $I_{0}(V)=\{0\}$ where 0 denotes the linear map with domain $\{0\}$ in $V$, and this map acts as a zero for the semigroup $I(V)$.

For arbitrary finite sets $X$, Yang [10] described all maximal inverse subsemigroups of each ideal of $I(X)$ in terms of the maximal subgroups of $G(X)$, the symmetric group on $X$, and all of these are known. Here we do the same for $I(V)$ where $V$ is any finitedimensional vector space over any field $F$, although all maximal subgroups of $G L(n, F)$, the general linear group of degree $n$ over $F$, are not known at present. As stated in [11] 
p. 162, "Currently, there is a fairly rich collection of examples of maximal subgroups of the general linear group. However, it is unclear ... how to find a description of all of them."

For example, in [1] Theorem 7, Borevich states that, if $n \geq 2$ and $F$ is any field with $|F| \geq 7$, then all maximal subgroups of $G L(n, F)$ that contain the group of diagonal matrices over $F$ are known. On the other hand, we recall that $Z(G)$, the centre of $G=G L(n, F)$, is the set of (non-zero) scalar matrices in $G$ and that, when $F=G F(q)$ is finite, $P G L(n, q)=G L(n, q) / Z(G)$ is the projective general linear group over $F$. It can be shown that, for $3 \leq n \leq 12, P G L(n, q)$ satisfies the criteria of [6] Theorem 1.2.2 and so all of its maximal subgroups are known. From this we deduce that, if $3 \leq n \leq 12$, then all subgroups of $G L(n, q)$ which are maximal and contain all the scalar matrices are known. However, simple examples show that a maximal subgroup of $G L(n, q)$ need not contain all the scalar matrices; and moreover, if one contains all the scalar matrices, then it need not contain all the diagonal matrices.

\section{Preliminary notation and results}

Throughout this paper, $V$ is a vector space with finite dimension $n$.

As an abbreviation, we write a subset $\left\{e_{i}: i \in I\right\}$ of $V$ as $\left\{e_{i}\right\}$, letting the subscript denote an (unspecified) index set $I$ (this is comparable with [2] Vol. 2, p. 241 and [7] Vol. 1, p. 51). The subspace $U$ of $V$ generated by a linearly independent subset $\left\{e_{i}\right\}$ of $V$ is denoted by $\left\langle e_{i}\right\rangle$, and we write $\operatorname{dim} U=|I|$.

Often it is necessary to construct some $\alpha \in I(V)$ by first choosing linearly independent subsets $\left\{e_{i}\right\}$ and $\left\{u_{i}\right\}$ of $V$, and then letting $e_{i} \alpha=u_{i}$ for each $i \in I$ and extending this action by linearity to the whole of $\operatorname{dom} \alpha=\left\langle e_{i}\right\rangle$. To abbreviate matters, we simply say, given $\left\{e_{i}\right\}$ and $\left\{u_{i}\right\}$ within context, that $\alpha \in I(V)$ is defined by letting

$$
\alpha=\left(\begin{array}{l}
e_{i} \\
u_{i}
\end{array}\right) .
$$

Similar notation for $I(X)$ is now standard: for example, see [9]. However, for simplicity, if $X$ is a set and $a, b \in X$, we write $a_{b}$ for the map in $I(X)$ with domain $\{a\}$ and range $\{b\}$.

Following [2] Vol. 1, p. 57, Exercise 6 (compare [8] section 3, p. 427, for endomorphisms of an independence algebra), it is easy to see that $\alpha \mathcal{D} \beta$ in $I(V)$ if and only if rank $\alpha=$ $\operatorname{rank} \beta$ (that is, $\mathcal{D}=\mathcal{J}$ ). Consequently, we denote each $\mathcal{D}$-class of $I(V)$ by

$$
D_{r}=\{\alpha \in I(V): \operatorname{rank} \alpha=r\}
$$


where $0 \leq r \leq n$ (when necessary, we use $D_{r}(V)$ to emphasise our use of linear maps). It is also easy to see that the idempotents of $I(V)$ are precisely the identity maps $\operatorname{id}_{A}$ whose domains $A$ are subspaces of $V$. Similar facts and notation are well-known for $I(X)$ : compare [9] pp. 309-310. In particular, we use $D_{r}(X)$ (or simply $D_{r}$ within context) to denote the $\mathcal{D}$-class of $I(X)$ consisting of all elements of $I(X)$ with rank $r$ where $0 \leq r \leq|X|$.

We begin by showing that $I(X)$ and $I(V)$ are never isomorphic if $X$ is a set and $V$ is a vector space over a field $F$ where $|X| \geq 1, \operatorname{dim} V \geq 1$ and $|F| \geq 3$ (this question was not considered in [8]). For, if such an isomorphism exists, then $I_{1}(X)$ is isomorphic to $I_{1}(V)$ since these are the smallest non-zero ideals of $I(X)$ and $I(V)$, respectively. But this is impossible since every element of $I_{1}(X)$ is either an idempotent or a nilpotent of index 2, whereas if $k \neq 0,1$ in $F$ and $u \neq 0$ in $V$ then $I_{1}(V)$ contains an element $\alpha$ with domain $\langle u\rangle$ such that $\alpha: u \mapsto k u$, and this is neither idempotent nor nilpotent.

To handle the case when $|F|=2$, we suppose $|X| \geq 3$ and assert that $I(X)$ satisfies:

$$
\begin{aligned}
\text { if } \alpha \in D_{2}(X) & \text { where } \alpha^{2} \text { is not idempotent and } \alpha \gamma, \alpha^{2} \gamma \text { are non-zero } \\
& \text { for some idempotent } \gamma \in D_{1}(X) \text {, then } \gamma \alpha=\emptyset .
\end{aligned}
$$

To see this, suppose $\alpha \in D_{2}(X)$ satisfies the initial condition and write

$$
\alpha=\left(\begin{array}{ll}
a & b \\
x & y
\end{array}\right) \text {. }
$$

Without loss of generality, let $\gamma=x_{x}$. If $x=a$ then $\alpha^{2}=a_{a}$ (if $\left.b \neq y\right)$ or $\alpha^{2}=\operatorname{id}_{\{a, b\}}$ (if $b=y$ ), contradicting our supposition that $\alpha^{2}$ is not idempotent. On the other hand, if $x=b$ then $y \neq b$ (since $\alpha$ is injective) and $y \neq a$ (since $\alpha^{2}$ is not idempotent). Hence $\alpha^{2}=a_{y}$ and so $\alpha^{2} \gamma=\emptyset$, another contradiction. Therefore, $x \neq a, b$ and so $\gamma \alpha=\emptyset$. Finally, note that $(*)$ is not vacuous in $I(X)$ if $|X| \geq 3$, since the map $b \rightarrow a \rightarrow x$, where $x \neq a, b$, satisfies $(*)$.

Clearly, property $(*)$ will be preserved under an isomorphism $\varphi$ from $I(X)$ onto $I(V)$. In fact, $\varphi$ will map the $\mathcal{D}$-classes of $I(X)$ onto the $\mathcal{D}$-classes of $I(V)$ in an obvious way. However, $I(V)$ does not satisfy $(*)$ if $\operatorname{dim} V \geq 3$ and $|F|=2$. For, in this case, if $a, b$ are two non-zero vectors in $V$ then they are linearly independent, and the same is true for $\{b, a+b\}$. Let

$$
\alpha=\left(\begin{array}{cc}
a & b \\
b & a+b
\end{array}\right) \quad \text { and } \quad \alpha^{2}=\left(\begin{array}{cc}
a & b \\
a+b & a
\end{array}\right)
$$

be linear maps defined and obtained as shown, and observe that $\alpha^{2}$ is not idempotent. Moreover, if $c=a+b$ and $\gamma \in D_{1}(V)$ is the idempotent with domain $\langle c\rangle$, then $\alpha \gamma$ and 
$\alpha^{2} \gamma$ are non-zero. However, $\gamma \alpha$ is also non-zero, so property $(*)$ fails to hold in $I(V)$. Therefore, $I(X)$ and $I(V)$ are not isomorphic when $|X| \geq 3, \operatorname{dim} V \geq 3$ and $|F|=2$. Thus we have proved most of the following result.

Theorem 1. The semigroups $I(X)$ and $I(V)$ are isomorphic only when $|X|=1=$ $\operatorname{dim} V$ and $|F|=2$.

Proof. For the remaining cases, we suppose $1<|X|=n<\aleph_{0}, 1<\operatorname{dim} V=m<\aleph_{0}$ and $|F|=2$. Clearly, the above discussion covers almost all of these situations but the argument we use here gives a more direct proof in the finite case. In fact, since $V$ has finite dimension $m$ over the field $F$, we know $V \cong F^{m}$ (as vector spaces) and so $|V|=2^{m}$. Also, the ideals of $I(X)$ form a chain with length $n+1$; and we have a similar result for $I(V)$ : its ideals form a chain with length $m+1$. Hence, if $I(X)$ and $I(V)$ are isomorphic then $n=m$ and, as mentioned above, $I_{1}(X)$ is isomorphic to $I_{1}(V)$. But the non-zero elements of $I_{1}(X)$ are the maps $a_{b}$ where $a, b \in X$, hence $\left|I_{1}(X)\right|=n^{2}+1$. And similarly, since $|F|=2$, the non-zero elements of $I_{1}(V)$ are the maps $u \mapsto v$ where $u, v$ are non-zero elements in $V$. Hence, if $m=n$ then $\left|I_{1}(V)\right|=\left(2^{n}-1\right)^{2}+1=2^{n+1}\left(2^{n-1}-1\right)+2$ and, since $2^{n+1}>n^{2}+1$ for every $n \geq 2$, it follows that $\left|I_{1}(V)\right|>\left|I_{1}(X)\right|$. Finally, observe that if $|X|=1=\operatorname{dim} V$ and $|F|=2$, then $I(X)$ and $I(V)$ are simply the group of order 1 with a zero adjoined.

In view of the above, it is interesting to give a linear version of the Vagner-Preston Theorem: our proof closely follows that of [2] Vol. 1, Theorem 1.20.

Theorem 2. Any inverse semigroup can be embedded in $I(V)$ for some vector space $V$.

Proof. Let $S$ be an inverse semigroup with $|S|=k$ (finite or infinite) and write $S=\left\{a_{i}\right\}$ with $|I|=k$. Let $F$ be any field and let $F_{i}$ be a copy of $F$ for each $i \in I$. As in [5] p. 182, Remark (c), we let $V$ be the vector space $\sum F_{i}$ over $F$ whose basis can be identified in a natural way with $\left\{a_{i}\right\}$ : that is, $\sum F_{i}$ is the set of all $\left(r_{i}\right)_{i \in I}$ where $r_{i} \in F_{i}$ and at most finitely many $r_{i}$ are non-zero.

Let $x \in S$ and suppose $\rho_{x}$ is the partial map with domain $S x^{-1}=S x x^{-1}$ such that $a \rho_{x}=a x\left(a \in S x^{-1}\right)$. The range of $\rho_{x}$ is $S x^{-1} x=S x$ and, in fact, $\rho_{x}$ is one-to-one: if $a, b \in S x^{-1}$ and $a \rho_{x}=b \rho_{x}$, then there exist $j, k \in I$ such that $a=a_{j} x^{-1}$ and $b=a_{k} x^{-1}$, so $a=a_{j} x^{-1}=a_{j} x^{-1} x x^{-1}=a_{k} x^{-1} x x^{-1}=a_{k} x^{-1}=b$. Since $S x x^{-1}$ is a subset of $S$, it is linearly independent. Hence $\rho_{x}$ can be extended by linearity to an injective linear map $\rho_{x}:\left\langle a_{i} x x^{-1}\right\rangle \rightarrow V$ with range $\left\langle a_{i} x\right\rangle$. Since $\left\{a_{i} x\right\}$ and $\left\{a_{i} x x^{-1}\right\}$ are bases for the 
domain of $\rho_{x}$, we can display $\rho_{x}$ as an element of $I(V)$ in two different ways.

$$
\rho_{x}=\left(\begin{array}{c}
a_{i} x^{-1} \\
a_{i} x^{-1} x
\end{array}\right) \quad \text { and } \quad \rho_{x}=\left(\begin{array}{c}
a_{i} x x^{-1} \\
a_{i} x
\end{array}\right) .
$$

We assert that $\phi: S \rightarrow I(V), x \mapsto \rho_{x}$, is an injective homomorphism.

Let $x, y \in S$ and suppose $x \phi=y \phi$, that is, $\rho_{x}=\rho_{y}$. Then, $\left\langle a_{i} x x^{-1}\right\rangle=\operatorname{dom} \rho_{x}=$ $\operatorname{dom} \rho_{y}=\left\langle a_{i} y y^{-1}\right\rangle$ and $a \rho_{x}=a \rho_{y}$ for all $a \in \operatorname{dom} \rho_{x}$. Since $x^{-1} \in S x x^{-1}=\left\{a_{i} x x^{-1}\right\}$, it follows that $x^{-1} x=x^{-1} \rho_{x}=x^{-1} \rho_{y}=x^{-1} y$. On the other hand, $x x^{-1} \in\left\{a_{i} x^{-1}\right\} \subseteq$ $\left\langle a_{i} x^{-1}\right\rangle=\left\langle a_{i} y^{-1}\right\rangle$ and so $x x^{-1}=\sum r_{i}\left(a_{i} y^{-1}\right)$ for some scalars $r_{i} \in F$. Since $\left\{x x^{-1}\right\} \cup$ $\left\{a_{i} y^{-1}\right\} \subseteq S$ and $S$ is linearly independent, this implies that there exists some $i_{0} \in I$ such that $r_{i_{0}}=1$ and $x x^{-1}=a_{i_{0}} y^{-1}$. Similarly we find that $y y^{-1}=a_{i_{1}} x^{-1}$ for some $i_{1} \in I$. Therefore, since idempotents commute in inverse semigroups, we have:

$$
\begin{aligned}
x x^{-1}=a_{i_{0}} y^{-1} & =a_{i_{0}} y^{-1} y y^{-1} \\
& =x x^{-1} y y^{-1}=y y^{-1} x x^{-1}=a_{i_{1}} x^{-1} x x^{-1}=a_{i_{1}} x^{-1}=y y^{-1} .
\end{aligned}
$$

Hence, $x=x x^{-1} x=x x^{-1} y=y y^{-1} y=y$.

To see that $\phi$ is a homomorphism, we let $x, y \in S$ and recall that $\rho_{x}, \rho_{y}$ are defined in $I(V)$ by

$$
\rho_{x}=\left(\begin{array}{c}
a_{i} x^{-1} \\
a_{i} x^{-1} x
\end{array}\right), \quad \rho_{y}=\left(\begin{array}{c}
a_{i} y y^{-1} \\
a_{i} y
\end{array}\right) .
$$

It is easy to see that $\rho_{x}^{-1}=\rho_{x^{-1}}$. Since $\left\{a_{i} x^{-1} x\right\} \cap\left\{a_{i} y y^{-1}\right\}=S x^{-1} x \cap S y y^{-1}=$ $S x^{-1} x y y^{-1}=S x y y^{-1}=\left\{a_{i} x y y^{-1}\right\}$, it follows that

$$
\rho_{x} \rho_{y}=\left(\begin{array}{c}
a_{i} x y y^{-1} x^{-1} \\
a_{i} x y y^{-1} y
\end{array}\right)=\left(\begin{array}{c}
a_{i}(x y)(x y)^{-1} \\
a_{i}(x y)
\end{array}\right)=\rho_{x y} .
$$

Hence $(x y) \phi=x \phi y \phi$.

\section{Maximal inverse subsemigroups of $I(V)$}

As in [10], to describe the maximal inverse subsemigroups of $I(V)$, we begin with two Lemmas: the second of these is comparable with [3] Theorem 3.1 (we let $G_{n}$ denote the group of all invertible linear transformations of $V$ ).

Lemma 1. For $1 \leq r \leq n-2, D_{r} \subseteq D_{r+1} D_{r+1}$.

Proof. Let $\alpha \in D_{r}$ and choose bases $\left\{a_{1}, \ldots, a_{r}\right\}$ and $\left\{b_{1}, \ldots, b_{r}\right\}$ for dom $\alpha$ and ran $\alpha$ respectively, where $a_{i} \alpha=b_{i}$ for each $i$. Since $r \leq n-2$, there exist $u_{0}, u_{1} \in V$ such 
that $\left\{a_{1}, \ldots, a_{r}, u_{0}, u_{1}\right\}$ is linearly independent; and likewise $\left\{b_{1}, \ldots, b_{r}, v_{1}\right\}$ is linearly independent for some $v_{1} \in V$. Then we have

$$
\alpha=\left(\begin{array}{ccc}
a_{1} & \ldots & a_{r} \\
b_{1} & \ldots & b_{r}
\end{array}\right)=\left(\begin{array}{llll}
a_{1} & \ldots & a_{r} & u_{0} \\
a_{1} & \ldots & a_{r} & u_{0}
\end{array}\right) \circ\left(\begin{array}{cccc}
a_{1} & \ldots & a_{r} & u_{1} \\
b_{1} & \ldots & b_{r} & v_{1}
\end{array}\right)
$$

where the two mappings on the right can be suitably extended by linearity to become elements of $D_{r+1}$.

Lemma 2. If $\alpha \in D_{n-1}$ then $I(V)=\left\langle\alpha, G_{n}\right\rangle$.

Proof. Suppose $a_{1}, \ldots, a_{n-1}$ and $b_{1}, \ldots, b_{n-1}$ are bases for dom $\alpha$ and ran $\alpha$ respectively, and assume that

$$
\alpha=\left(\begin{array}{ccc}
a_{1} & \ldots & a_{n-1} \\
b_{1} & \ldots & b_{n-1}
\end{array}\right)
$$

To show that $\left\langle\alpha, G_{n}\right\rangle$ contains a mapping with rank $n-2$, we consider two cases.

Case 1. Suppose dom $\alpha=\operatorname{ran} \alpha=A$ say. Then there exists $u \notin A$ and $g \in G_{n}$ defined by

$$
g=\left(\begin{array}{ccccc}
b_{1} & \ldots & b_{n-2} & b_{n-1} & u \\
b_{1} & \ldots & b_{n-2} & u & b_{n-1}
\end{array}\right)
$$

Since $\left\langle b_{1}, \ldots, b_{n-2}\right\rangle \subseteq \operatorname{dom} \alpha$ and $\alpha$ is injective, we obtain

$$
\alpha g \cdot \alpha=\left(\begin{array}{cccc}
a_{1} & \ldots & a_{n-2} & a_{n-1} \\
b_{1} & \ldots & b_{n-2} & u
\end{array}\right) \circ\left(\begin{array}{cccc}
a_{1} & \ldots & a_{n-2} & a_{n-1} \\
b_{1} & \ldots & b_{n-2} & b_{n-1}
\end{array}\right)=\left(\begin{array}{ccc}
a_{1} & \ldots & a_{n-2} \\
c_{1} & \ldots & c_{n-2}
\end{array}\right)
$$

for some linearly independent $c_{1}, \ldots, c_{n-2} \in V$.

Case 2. Suppose $A=\operatorname{dom} \alpha \neq \operatorname{ran} \alpha=B$. Since $A$ and $B$ have the same finite dimension, this implies $A \nsubseteq B$. Choose $u \in A \backslash B$, let $\left\{c_{1}, \ldots, c_{n-2}, u\right\}$ be a basis for $A$ and write

$$
\alpha=\left(\begin{array}{cccc}
c_{1} & \ldots & c_{n-2} & u \\
d_{1} & \ldots & d_{n-2} & v
\end{array}\right)
$$

where $\left\{d_{1}, \ldots, d_{n-2}, v\right\}$ is a basis for $B$ (hence $\left\{d_{1}, \ldots, d_{n-2}, v, u\right\}$ is a basis for $V$ ). Choose $w \notin A$ and define $g \in G_{n}$ by

$$
g=\left(\begin{array}{ccccc}
d_{1} & \ldots & d_{n-2} & v & u \\
c_{1} & \ldots & c_{n-2} & u & w
\end{array}\right) .
$$

Then

$$
\alpha g=\left(\begin{array}{cccc}
c_{1} & \ldots & c_{n-2} & u \\
c_{1} & \ldots & c_{n-2} & u
\end{array}\right)
$$


and, as in Case 1, we can use this to obtain a mapping in $\left\langle\alpha, G_{n}\right\rangle$ with rank $n-2$.

Clearly we can repeat the above process to obtain an element of $\left\langle\alpha, G_{n}\right\rangle$ with rank $r$ where $0 \leq r \leq n-1$. Then if

$$
\beta=\left(\begin{array}{ccc}
e_{1} & \ldots & e_{r} \\
f_{1} & \ldots & f_{r}
\end{array}\right)
$$

is any element of $\left\langle\alpha, G_{n}\right\rangle$ with rank $r$, we can expand $\operatorname{dom} \beta$ and $\operatorname{ran} \beta$ to bases

$$
\left\{e_{1}, \ldots, e_{r}, e_{r+1}, \ldots, e_{n}\right\} \text { and }\left\{f_{1}, \ldots, f_{r}, f_{r+1}, \ldots, f_{n}\right\}
$$

for $V$. Also, if $\left\{u_{1}, \ldots, u_{r}\right\}$ and $\left\{v_{1}, \ldots, v_{r}\right\}$ are any linearly independent subsets of $V$, we can expand them to bases $\left\{u_{1}, \ldots, u_{r}, u_{r+1}, \ldots, u_{n}\right\}$ and $\left\{v_{1}, \ldots, v_{r}, v_{r+1}, \ldots, v_{n}\right\}$ for $V$ and then define $g, h \in G_{n}$ by $u_{i} g=e_{i}$ and $f_{i} h=v_{i}$ for each $i$. Then

$$
g \beta h=\left(\begin{array}{ccc}
u_{1} & \ldots & u_{r} \\
v_{1} & \ldots & v_{r}
\end{array}\right) \in\left\langle\alpha, G_{n}\right\rangle .
$$

Since this is an arbitrary element of $I(V)$ with $\operatorname{rank} r$, we conclude that $I(V)=\left\langle\alpha, G_{n}\right\rangle$.

The proof of the next result follows that of [10] Theorem 2.3. For convenience, here we write $I_{r}(V)$ more simply as $I_{r}$.

Theorem 3. If $\operatorname{dim} V=n<\aleph_{0}$, the maximal inverse subsemigroups of $I(V)$ are precisely the following sets.

(a) $I_{n-2} \cup G_{n}$,

(b) $I_{n-1} \cup H$, where $H$ is a maximal subgroup of $G_{n}$.

Proof. Clearly, $I_{n-1} \cup H$ is a maximal inverse subsemigroup of $I(V)$ in (b). Also, $I_{n-2} \cup G_{n}$ is an inverse semigroup. In fact, if $I_{n-2} \cup G_{n} \varsubsetneqq T \subseteq I(V)$ for some inverse semigroup $T$ then $T \cap D_{n-1} \neq \emptyset$ and so Lemma 2 implies $T=I(V)$.

Conversely, suppose $S$ is a maximal inverse subsemigroup of $I(V)$. Then $S \cap G_{n} \neq \emptyset$ : otherwise, $S \subseteq I_{n-1} \varsubsetneqq I_{n-1} \cup H$ for any maximal subgroup $H$ of $G_{n}$, contradicting the maximality of $S$.

Case 1. $S \cap G_{n}=G_{n}$. This means $G_{n} \subseteq S$ and hence $S \cap D_{n-1}=\emptyset$ : otherwise, $\left\langle\alpha, G_{n}\right\rangle \subseteq S$ for some $\alpha \in D_{n-1}$ and then Lemma 2 implies $S=I(V)$, a contradiction as before. Consequently, $S \subseteq I_{n-2} \cup G_{n}$ which is an inverse subsemigroup of $I(V)$, hence $S=I_{n-2} \cup G_{n}$ by the maximality of $S$. 
Case 2. $S \cap G_{n}=H \neq G_{n}$. Note that $H$ is a group since $S$ is inverse (that is, $\alpha \in H$ implies $\alpha^{-1} \in H$ and clearly $H$ is a semigroup). Now $S \subseteq I_{n-1} \cup H$ which is a proper inverse subsemigroup of $I(V)$, hence $S=I_{n-1} \cup H$. Moreover, if $H \varsubsetneqq H^{\prime} \varsubsetneqq G_{n}$ for some subgroup $H^{\prime}$ of $G_{n}$, we have

$$
S=I_{n-1} \cup H \varsubsetneqq I_{n-1} \cup H^{\prime} \varsubsetneqq I(V),
$$

which contradicts the maximality of $S$. Hence, $H$ is a maximal subgroup of $G_{n}$.

\section{Maximal inverse subsemigroups of $I_{r}$}

Given a group $G$, two arbitrary sets $J$ and $\Lambda$, and an arbitrary but fixed $\Lambda \times J$ matrix $P=\left(p_{j \lambda}\right)$ over $G^{0}$, the group with zero adjoined, then the Rees $J \times \Lambda$ matrix semigroup over $G^{0}$ with sandwich matrix $P$ is the semigroup $\mathcal{M}^{0}(G ; J, \Lambda ; P)$ of all triples $(j, a, \lambda)$ with $a \in G^{0}, j \in J$ and $\lambda \in \Lambda$ and a product defined as follows: given $(j, a, \lambda),(i, b, \mu) \in$ $\mathcal{M}^{0}(G ; J, \Lambda ; P),(j, a, \lambda)(i, b, \mu)=\left(j, a p_{\lambda i} b, \mu\right)$ (compare [2] Vol. 1, p. 88: we use $J$ instead of the more usual $I$ to avoid any confusion with earlier notation; and we use $(j, a, \lambda)$ rather than $(a, j, \lambda)$ to maintain some uniformity with the notation in [10]). If $J=\Lambda$ and $P$ is the identity $J \times J$ matrix over $G^{0}$, then we say the semigroup $\mathcal{M}^{0}(G ; J, \Lambda ; P)$ is a Brandt semigroup and we denote it more simply by $B(G, J)$. From [2] Theorem 3.9, the semigroup $B(G, J)$ is a completely 0-simple inverse semigroup. Also, by [2] Lemma 3.2, Green's relations on $B(G, J)$ are characterised as follows: given $(j, a, \lambda),(i, b, \mu)$ in $B(G, J)$, we have $(j, a, \lambda) \mathcal{L}(i, b, \mu)$ if and only if $\lambda=\mu$; and $(j, a, \lambda) \mathcal{R}(i, b, \mu)$ if and only if $j=i$.

As in [10], if $S$ is an inverse semigroup and $A$ is a non-empty subset of $S$, then we write $\langle\langle A\rangle\rangle$ for the inverse subsemigroup of $S$ generated by $A$.

In [10] section 3, Yang first determines all maximal inverse subsemigroups of the Brandt semigroup $B(G, J)$ where $G$ is a finite group and $J=\{1, \ldots, m\}$. He requires two preliminary results ([10] Lemmas 3.2 and 3.3) to do this, and both of these depend on the following result. However, Yang's version of this result covers only the finite case. Since we need to know all maximal inverse subsemigroups of $B(G, J)$ where $G$ is an arbitrary group with identity $e$ and $J$ is an arbitrary index set, we include a proof of the following result for clarity and completeness (for the reason why we need such generality, see the discussion after Lemma 6 below). Here, we write $B^{A}$ for the set of all maps $g: A \rightarrow B$, and represent $g$ by $\left[g_{a}\right]$ (that is, $A$ acts as an index set for the images of $g$ in $B$, hence the $g_{a}$ 's are not necessarily distinct).

Lemma 3. For each $i \in J$, let $H_{i i}$ denote the $\mathcal{H}$-class of $B(G, J)$ containing the 
element $(i, e, i)$, put $K_{i}=J \backslash\{i\}$ and let $\left[g_{j}\right] \in G^{K_{i}}$. Then,

$$
B(G, J)=\left\langle\left\langle H_{i i} \cup\left\{\left(i, g_{j}, j\right): j \in K_{i}\right\}\right\rangle\right\rangle .
$$

Proof. Let $(j, g, k) \in B(G, J)$. If $j=k=i$, then $(j, g, k) \in H_{i i}$. If $j=i$ and $k \neq i$, then $(j, g, k)=\left(i, g g_{k}^{-1}, i\right)\left(i, g_{k}, k\right)$ where $\left(i, g g_{k}^{-1}, i\right) \in H_{i i}$; and similarly, if $j \neq i$ and $k=i$, then we can write $(j, g, k)$ as $\left(i, g_{j}, j\right)^{-1}\left(i, g_{j} g, i\right)$. If $j \neq i$ and $k \neq i$, then $(j, g, k)=\left(i, g_{j}, j\right)^{-1}\left(i, g_{j} g g_{k}^{-1}, i\right)\left(i, g_{k}, k\right)$. Therefore, each $(j, g, k)$ can be written as a finite product of elements of $H_{i i} \cup\left\{\left(i, g_{j}, j\right): j \in K_{i}\right\}$ and their inverses, and the result follows.

The proofs of [10] Lemmas 3.2 and 3.3 are stated for finite groups and finite index sets and, in some places, the notation and argument is not entirely clear (for example, in [10] Lemma 3.2, $I=\{1, \ldots, m\}$ and $g_{1}, g_{2}, \ldots, g_{m} \in G$ where $g_{1}=e$, the identity of the group $G$, all of which appears to require $G$ to have order at least $m$, but this is not intended). For this reason, and since we must deal with arbitrary groups and arbitrary index sets, we prove the following Lemmas in detail, but use the basic idea of Yang's proofs to do so.

The first Lemma determines some maximal inverse subsemigroups of $B(G, J)$.

Lemma 4. Let $H$ be a maximal subgroup of $G$ and let $1 \in J$. Put $H_{11}^{*}=\{(1, h, 1) \in$ $B(G, J): h \in H\}$. Let $\left[g_{j}\right] \in G^{J}$ with $g_{1}=e$ and let

$$
B_{H}=\left\langle\left\langle H_{11}^{*} \cup\left\{\left(1, g_{j}, j\right): j \in J\right\}\right\rangle\right\rangle .
$$

Then $B_{H}$ is isomorphic to the Brandt semigroup $B(H, J)$ and it is a maximal inverse subsemigroup of $B(G, J)$.

Proof. Let $H_{i j}$ denote the $\mathcal{H}$-class of $B(G, J)$ containing the element $(i, e, j)$, that is: $H_{i j}=\{(i, g, j): g \in G\}$, and write $\left\{\left(i, g_{i}^{-1} h g_{j}, j\right): h \in H\right\}$ as $\left(i, g_{i}^{-1} H g_{j}, j\right)$. Consider the set $H_{i j} \cap B_{H}$ and let $(i, g, j) \in B_{H}$. Then, $(i, g, j)$ can be written as a finite product, say $y_{1} \cdots y_{r}$, of elements of $H_{11}^{*} \cup\left\{\left(1, g_{j}, j\right): j \in J\right\}$ and their inverses. Clearly, $y_{1}=\left(i, g_{i}^{-1}, 1\right)$ and $y_{r}=\left(1, g_{j}, j\right)$ and so $y_{2} \cdots y_{r-1}=\left(1, g_{i} g g_{j}^{-1}, 1\right)$ (for example, $(i, g, j)=y_{1} y_{2} \cdots y_{r}$ and $y_{1}=\left(i, g_{i}^{-1}, 1\right)$ imply $\left(1, g_{i} g, j\right)=\left(1, g_{i}, i\right)(i, g, j)=(1, e, 1)$. $\left.y_{2} \cdots y_{r}=y_{2} \cdots y_{r}\right)$. Now, if $y_{2}=\left(1, g_{k}, k\right)$ for some $k \in J \backslash\{1\}$, then $y_{3}$ must equal $\left(k, g_{k}^{-1}, 1\right)$, so $y_{2} y_{3}=(1, e, 1)$ and we then consider $y_{4} \cdots y_{r-1}$; alternatively, if $y_{2}=\left(1, h_{2}, 1\right)$ for some $h_{2} \in H$, then $y_{3} \cdots y_{r-1}$ equals $\left(1, g^{\prime}, 1\right)$ for some $g^{\prime} \in$ $G$ and we repeat this argument by starting with $y_{3}$. It follows that $y_{2} \cdots y_{r-1} \in$ $H_{11}^{*}$ and there exists some $h$ in $H$ such that $y_{2} \cdots y_{r-1}=(1, h, 1)$. Thus $(i, g, j)=$ $\left(i, g_{i}^{-1}, 1\right)(1, h, 1)\left(1, g_{j}, j\right)=\left(i, g_{i}^{-1} h g_{j}, j\right) \in\left(i, g_{i}^{-1} H g_{j}, j\right)$. Conversely, given $h \in H$, we 
have $\left(i, g_{i}^{-1} h g_{j}, j\right)=\left(i, g_{i}^{-1}, 1\right)(1, h, 1)\left(1, g_{j}, j\right) \in B_{H}$ and so $\left(i, g_{i}^{-1} h g_{j}, j\right) \in H_{i j} \cap B_{H}$. Hence, $H_{i j} \cap B_{H}=\left(i, g_{i}^{-1} H g_{j}, j\right)$.

Now define a mapping $\theta: B(H, J) \rightarrow B_{H}$ as follows: $0 \theta=0$ and $(i, h, j) \theta=$ $\left(i, g_{i}^{-1} h g_{j}, j\right)$. We assert that $\theta$ is an isomorphism from $B(H, J)$ onto $B_{H}$.

To see that $\theta$ is a morphism, let $\left(i, h_{1}, j\right),\left(r, h_{2}, s\right) \in B(H, J)$. If $j \neq r$, then

$$
\left(i, h_{1}, j\right) \theta\left(r, h_{2}, s\right) \theta=\left(i, g_{i}^{-1} h_{1} g_{j}, j\right)\left(r, g_{r}^{-1} h_{2} g_{s}, s\right)=0=0 \theta=\left[\left(i, h_{1}, j\right)\left(r, h_{2}, s\right)\right] \theta .
$$

On the other hand, if $j=r$, then

$$
\begin{aligned}
\left(i, h_{1}, j\right) \theta\left(r, h_{2}, s\right) \theta & =\left(i, g_{i}^{-1} h_{1} g_{j}, j\right)\left(j, g_{j}^{-1} h_{2} g_{s}, s\right)=\left(i, g_{i}^{-1} h_{1} g_{j} g_{j}^{-1} h_{2} g_{s}, s\right) \\
& =\left(i, g_{i}^{-1} h_{1} h_{2} g_{s}, s\right)=\left(i, h_{1} h_{2}, s\right) \theta \\
& =\left[\left(i, h_{1}, j\right)\left(j, h_{2}, s\right)\right] \theta=\left[\left(i, h_{1}, j\right)\left(r, h_{2}, s\right)\right] \theta .
\end{aligned}
$$

Clearly, given $x \in B(H, J), x \theta=0$ if and only if $x=0$. If $\left(i, h_{1}, j\right)$ and $\left(r, h_{2}, s\right)$ are such that $\left(i, h_{1}, j\right) \theta=\left(r, h_{2}, s\right) \theta$, then $i=r, j=s$ and $g_{i}^{-1} h_{1} g_{j}=g_{i}^{-1} h_{2} g_{j}$, and so $h_{1}=h_{2}$. Hence, $\left(i, h_{1}, j\right)=\left(r, h_{2}, s\right)$ and $\theta$ is one-to-one.

Next, let $x \in B_{H} \backslash\{0\}$. Since $x \in B(G, J)$, there exist $i, j \in J$ and $g \in G$ such that $x=(i, g, j)$. Thus, $x \in H_{i j} \cap B_{H}$ and so there is some $h$ in $H$ such that $x=$ $\left(i, g_{i}^{-1} h g_{j}, j\right)=(i, h, j) \theta$. Hence, $\theta$ is an isomorphism from $B(H, J)$ onto $B_{H}$.

To show that $B_{H}$ is maximal, it suffices to show that $B(H, J)$ is maximal. Let $S$ be an inverse subsemigroup of $B(G, J)$ properly containing $B(H, J)$. Then, there exists some $(i, a, j) \in S \backslash B(H, J)$. It follows that $a \in G \backslash H$ and, since $H$ is a maximal subgroup of $G$, we have $G=\langle\langle H \cup\{a\}\rangle\rangle$. Also,

$$
(1, a, 1)=(1, e, i)(i, a, j)(j, e, 1) \in B(H, J) \cdot S \cdot B(H, J) \subseteq S
$$

and so $\left(1, a^{-1}, 1\right)=(1, a, 1)^{-1} \in S$. Since $H$ is a group, every $g \in G$ can be written as a finite product, say $x_{1} \cdots x_{\ell}$, of elements of $H \cup\left\{a, a^{-1}\right\}$. Since $B(H, J) \subseteq S$, it follows that $\left(1, x_{t}, 1\right) \in S$ for every $t \in\{1, \ldots, \ell\}$, and so $(1, g, 1)=\left(1, x_{1}, 1\right) \cdots\left(1, x_{\ell}, 1\right) \in S$. Hence, $H_{11} \subseteq S$. From Lemma $3, B(G, J)=\left\langle\left\langle H_{11} \cup\left\{\left(1, g_{j}, j\right): j \in K_{1}\right\}\right\rangle\right\rangle$ for some $\left[g_{j}\right] \in G^{K_{1}}$ with $K_{1}=J \backslash\{1\}$. Let $j \in J$. Then, $\left(1, g_{j}, j\right)=\left(1, g_{j}, 1\right)(1, e, j) \in$ $S . B(H, J) \subseteq S$. Therefore, $B(G, J) \subseteq S$ and so $B(H, J)$ is maximal.

As in [10], a pair $\left(J_{1}, J_{2}\right)$ is said to be a two-partition of $J$ if $J=J_{1} \cup J_{2}$ and $J_{1} \cap J_{2}=$ $\emptyset$. The next Lemma determines another family of maximal inverse subsemigroups of $B(G, J)$.

Lemma 5. Let $\left(J_{1}, J_{2}\right)$ be a two-partition of $J$ and let $B_{J_{1} J_{2}}=B\left(G, J_{1}\right) \cup B\left(G, J_{2}\right)$. Then, $B_{J_{1} J_{2}}$ is a maximal inverse subsemigroup of $B(G, J)$. 
Proof. Clearly, $B\left(G, J_{1}\right) \cap B\left(G, J_{2}\right)=\{0\}$ and $B\left(G, J_{1}\right), B\left(G, J_{2}\right)$ are inverse subsemigroups of $B(G, J)$. Also, $\left(i_{1}, g, j_{1}\right)\left(i_{2}, g^{\prime}, j_{2}\right)=0$ for all $\left(i_{1}, g, j_{1}\right) \in B\left(G, J_{1}\right)$ and $\left(i_{2}, g^{\prime}, j_{2}\right) \in B\left(G, J_{2}\right)$. Thus, $B_{J_{1} J_{2}}$ is an inverse subsemigroup of $B(G, J)$.

To see that $B_{J_{1} J_{2}}$ is maximal, let $S$ be an inverse subsemigroup of $B(G, J)$ properly containing $B_{J_{1} J_{2}}$. Then, there exists $(i, b, j) \in S \backslash B_{J_{1} J_{2}}$. Since $S$ is an inverse semigroup, we have $\left(j, b^{-1}, i\right)=(i, b, j)^{-1} \in S$. Also, since $(i, b, j) \notin B_{J_{1} J_{2}}$, it follows that either $\left(i \in J_{1}\right.$ and $\left.j \in J_{2}\right)$ or $\left(i \in J_{2}\right.$ and $\left.j \in J_{1}\right)$. Hence, there exist $j_{1} \in J_{1}, a \in G$ and $j_{2} \in J_{2}$ such that $\left(j_{1}, a, j_{2}\right) \in S$. Let $R_{j_{1}}$ denote the $\mathcal{R}$-class of $B(G, J)$ containing $\left(j_{1}, a, j_{2}\right)$, that is, $R_{j_{1}}=\left\{\left(j_{1}, g, j\right): g \in G, j \in J\right\}$. Given $\left(j_{1}, g, j\right) \in R_{j_{1}}$, if $j \in J_{1}$, then $\left(j_{1}, g, j\right) \in B\left(G, J_{1}\right) \subseteq S$; and if $j \in J_{2}$, then $\left(j_{1}, g, j\right)=\left(j_{1}, a, j_{2}\right)\left(j_{2}, a^{-1} g, j\right) \in$ $S . B\left(G, J_{2}\right) \subseteq S$. Therefore, $R_{j_{1}} \subseteq S$. From Lemma 3, if $K_{j_{1}}=J \backslash\left\{j_{1}\right\}$, then there exists $\left[g_{j}\right] \in G^{K_{j_{1}}}$ such that $B(G, J)=\left\langle\left\langle H_{j_{1} j_{1}} \cup\left\{\left(j_{1}, g_{j}, j\right): j \in K_{j_{1}}\right\}\right\rangle\right\rangle$. Since $H_{j_{1} j_{1}} \cup\left\{\left(j_{1}, g_{j}, j\right): j \in K_{j_{1}}\right\} \subseteq R_{j_{1}} \subseteq S$ and $S$ is an inverse semigroup, it follows that $B(G, J) \subseteq S$, as required.

In essence, the next result is [10] Proposition 3.4, but in a more general setting, albeit with $J$ restricted to being an index set with at least two elements. Once again, we follow most of Yang's argument closely.

Theorem 4. Let $S$ be a subsemigroup of the Brandt semigroup $B(G, J)$, where $G$ is an arbitrary group with identity $e$ and $J$ is an index set with at least two elements. Then, $S$ is a maximal inverse subsemigroup of $B(G, J)$ if and only if $S$ is one of the following:

(a) $S=B\left(G, J_{1}\right) \cup B\left(G, J_{2}\right)$, where $\left(J_{1}, J_{2}\right)$ is a two-partition of $J$,

(b) $S=\left\langle\left\langle H_{11}^{*} \cup\left\{\left(1, g_{j}, j\right): j \in K_{1}\right\}\right\rangle\right\rangle$, where $1 \in J, K_{1}=J \backslash\{1\}, H_{11}^{*}=\{(1, h, 1) \in$ $B(G, J): h \in H\}, H$ is a maximal subgroup of $G$ and $\left[g_{j}\right] \in G^{K_{1}}$.

Proof. From Lemmas 4 and 5 , if $S$ is of type (a) or (b) then it is a maximal inverse subsemigroup of $B(G, J)$. Conversely, let $S$ be a maximal inverse subsemigroup of $B(G, J)$. We assert that $S \cap H_{j j} \neq \emptyset$ for each $j \in J$. For, suppose $S \cap H_{i i}=\emptyset$ for some $i \in J$. Then, $S \cap L_{i}=\emptyset$ where $L_{i}$ is the $\mathcal{L}$-class of $B(G, J)$ which contains the element $(i, e, i)$. For, if $S \cap L_{i} \neq \emptyset$, then there exist some $j \in J$ and $a \in G$ such that $(j, a, i) \in S$, and so $(i, e, i)=\left(i, a^{-1}, j\right)(j, a, i)=(j, a, i)^{-1}(j, a, i) \in S \cap H_{i i}$, a contradiction. Similarly, $S \cap R_{i}=\emptyset$ where $R_{i}$ denotes the $\mathcal{R}$-class of $B(G, J)$ containing $(i, e, i)$. Hence,

$$
S \subseteq B(G, J \backslash\{i\}) \varsubsetneqq B(G, J \backslash\{i\}) \cup H_{i i} \varsubsetneqq B(G, J)
$$


Clearly, $B(G, J \backslash\{i\}) \cup H_{i i}$ is an inverse subsemigroup of $B(G, J)$, which contradicts our assumption on $S$. Note that, in the above argument, we use the fact that $|J| \geq 2$.

We now consider two cases.

Case 1. Suppose $S \cap H_{11}=H_{11}$. Let $J_{1}=\left\{j \in J: S \cap H_{1 j} \neq \emptyset\right\}$ and $J_{2}=J \backslash J_{1}$, and note that $J_{1} \neq \emptyset$ by supposition. If $S \cap H_{1 j} \neq \emptyset$, then there exists some $a \in G$ such that $(1, a, j) \in S$, and so we have $(1, g, j)=\left(1, g a^{-1}, 1\right)(1, a, j) \in H_{11} . S \subseteq S$, for every $(1, g, j) \in H_{1 j}$. Hence, $S \cap H_{1 j}=H_{1 j}$ for each $j \in J_{1}$. Thus, given $(i, g, j) \in B\left(G, J_{1}\right)$, we have $S \cap H_{1 i}=H_{1 i}$ and $S \cap H_{1 j}=H_{1 j}$, and so $(i, g, j)=\left(1, g^{-1}, i\right)^{-1}(1, e, j) \in$ $H_{1 i}^{-1} \cdot H_{1 j} \subseteq S$. Therefore, $B\left(G, J_{1}\right) \subseteq S$.

Suppose there exist $j_{1} \in J_{1}, a \in G$ and $j_{2} \in J_{2}$ such that $\left(j_{1}, a, j_{2}\right) \in S$. Then, $\left(1, a, j_{2}\right)=\left(1, e, j_{1}\right)\left(j_{1}, a, j_{2}\right) \in H_{1 j_{1}} . S \subseteq S$, since $j_{1} \in J_{1}$ implies $S \cap H_{1 j_{1}} \neq \emptyset$ and so $H_{1 j_{1}} \subseteq S$ by a remark in the last paragraph. Hence $\left(1, a, j_{2}\right) \in S \cap H_{1 j_{2}}$, so this set is non-empty and therefore $j_{2} \in J_{1}$, a contradiction. Thus, since $S$ is inverse, given $j_{1} \in J_{1}, a \in G$ and $j_{2} \in J_{2}$, both $\left(j_{1}, a, j_{2}\right) \notin S$ and $\left(j_{2}, a, j_{1}\right) \notin S$. We then have $S \subseteq B\left(G, J_{1}\right) \cup B\left(G, J_{2}\right) \varsubsetneqq B(G, J)$. By maximality of $S$, it follows that $S=B\left(G, J_{1}\right) \cup B\left(G, J_{2}\right)$.

Case 2. Suppose $S \cap H_{11} \neq H_{11}$. Let $H_{11}^{*}=S \cap H_{11}$ and $H=\left\{h \in G:(1, h, 1) \in H_{11}^{*}\right\}$. We assert that $H$ is a proper subgroup of $G$. Since $S \cap H_{11} \neq \emptyset$, there exists $a \in G$ such that $(1, a, 1) \in S \cap H_{11}=H_{11}^{*}$. Then, $(1, e, 1)=(1, a, 1)(1, a, 1)^{-1} \in H_{11}^{*}$ and so $e \in H$. Now suppose $h_{1}, h_{2} \in H$. Then, $\left(1, h_{1}, 1\right),\left(1, h_{2}, 1\right) \in H_{11}^{*}$. Since $H_{11}^{*}$ is an inverse subsemigroup of $B(G, J)$, it follows that $\left(1, h_{1}, 1\right)\left(1, h_{2}, 1\right)^{-1}=\left(1, h_{1} h_{2}^{-1}, 1\right) \in$ $H_{11}^{*}$ and so $h_{1} h_{2}^{-1} \in H$. Since $S \cap H_{11} \neq H_{11}$, there exists some $g \in G$ such that $(1, g, 1) \notin S \cap H_{11}=H_{11}^{*}$, and so $g \notin H$ and $H \varsubsetneqq G$.

Still in Case 2, let $J^{*}=\left\{j \in J: S \cap H_{1 j} \neq \emptyset\right\}$ and choose $\left(1, g_{j}, j\right) \in S \cap H_{1 j}$ for each $j \in J^{*}$. Clearly, $\left\langle\left\langle H_{11}^{*} \cup\left\{\left(1, g_{j}, j\right): j \in J^{*}\right\}\right\rangle\right\rangle \subseteq S$. We assert that $J=J^{*}$. For, if $J \backslash J^{*} \neq \emptyset$, then $S \cap H_{1 i}=\emptyset$ for each $i \in J \backslash J^{*}$. Now suppose $(i, a, j) \in S$ with $i \notin J^{*}$ and $j \in J^{*}$. Then, $\left(1, g_{j} a^{-1}, i\right)=\left(1, g_{j}, j\right)\left(j, a^{-1}, i\right)=\left(1, g_{j}, j\right)(i, a, j)^{-1} \in S$ (since $S$ is inverse) which contradicts $S \cap H_{1 i}=\emptyset$. In other words, if $(i, a, j) \in S$ then either $\left(i \in J^{*}\right.$ and $\left.j \in J^{*}\right)$ or $\left(i \notin J^{*}\right.$ and $\left.j \notin J^{*}\right)$. Therefore, since $1 \in J^{*}$ (by a remark at the start of this proof) and $H_{11} \backslash S$ is non-empty (by assumption in this case), it follows that

$$
S \varsubsetneqq B\left(G, J^{*}\right) \cup B\left(G, J \backslash J^{*}\right) \varsubsetneqq B(G, J) .
$$

But $B\left(G, J^{*}\right) \cup B\left(G, J \backslash J^{*}\right)$ is an inverse subsemigroup of $B(G, J)$, so (1) contradicts the maximality of $S$. Therefore, $J=J^{*}$.

Next we see that $S=\left\langle\left\langle H_{11}^{*} \cup\left\{\left(1, g_{j}, j\right): j \in J\right\}\right\rangle\right\rangle$. For convenience, we let $g_{1}=e$ 
and for every $i$ and $j$ in $J$, we denote the subset $\left\{\left(i, g_{i}^{-1} h g_{j}, j\right): h \in H\right\}$ of $B(G, J)$ by $\left(i, g_{i}^{-1} H g_{j}, j\right)$. We assert that $S \cap H_{i j}=\left(i, g_{i}^{-1} H g_{j}, j\right)$. For, if $h \in H$, we have

$$
\left(i, g_{i}^{-1} h g_{j}, j\right)=\left(1, g_{i}, i\right)^{-1}(1, h, 1)\left(1, g_{j}, j\right) \in S \cdot H_{11}^{*} \cdot\left(S \cap H_{1 j}\right) \subseteq S
$$

and so $\left(i, g_{i}^{-1} h g_{j}, j\right) \in S \cap H_{i j}$. Conversely, let $(i, a, j) \in S \cap H_{i j}$. Then, $\left(1, g_{i} a g_{j}^{-1}, 1\right)=$ $\left(1, g_{i}, i\right)(i, a, j)\left(1, g_{j}, j\right)^{-1} \in S \cap H_{11}=H_{11}^{*}$ and so $g_{i} a g_{j}^{-1} \in H$. Thus, $(i, a, j)=$ $\left(i, g_{i}^{-1} h g_{j}, j\right)$ where $h=g_{i} a g_{j}^{-1} \in H$, and so $S \cap H_{i j}=\left(i, g_{i}^{-1} H g_{j}, j\right)$. Now, if $(i, a, j) \in$ $S$, then $(i, a, j)=\left(i, g_{i}^{-1} h g_{j}, j\right)=\left(1, g_{i}, i\right)^{-1}(1, h, 1)\left(1, g_{j}, j\right)$ for some $h \in H$, and so $(i, a, j)$ can be written as a finite product of elements of $H_{11}^{*} \cup\left\{\left(1, g_{j}, j\right): j \in J\right\}$ and inverses of such elements. Therefore, $S \subseteq\left\langle\left\langle H_{11}^{*} \cup\left\{\left(1, g_{j}, j\right): j \in J\right\}\right\rangle\right\rangle$, and equality follows from the containment observed earlier.

It remains to show that $H$ is a maximal subgroup of $G$. Suppose this is not true. Then, there exists a proper subgroup $K$ of $G$ which properly contains $H$. Let $K_{11}^{*}=$ $\{(1, k, 1): k \in K\}$. Clearly, $H_{11}^{*} \varsubsetneqq K_{11}^{*}$ and so $S$ is a proper subsemigroup of the inverse semigroup $\left\langle\left\langle K_{11}^{*} \cup\left\{\left(1, g_{j}, j\right): j \in J\right\}\right\rangle\right\rangle$, a contradiction. Therefore, $H$ is a maximal subgroup of $G$, and the result follows.

Now we turn our attention to the inverse semigroup $I_{r}=\{\alpha \in I(V)$ : rank $\alpha \leq r\}$ for $1 \leq r \leq n$ where $n=\operatorname{dim} V$. From Lemma 1 , the $\mathcal{D}$-class $D_{r}$ generates $I_{r}$ since, if $s \in\{1, \ldots, r-1\}$, then $D_{s} \subseteq D_{r}^{k}$ where $k=2^{r-s}$. Since $D_{r}=I_{r} \backslash I_{r-1}$ is a $\mathcal{D}$-class of $I(V)$, the principal factor $I_{r} / I_{r-1}$ is 0 -simple. Moreover, $I_{r} / I_{r-1}$ contains non-zero idempotents: namely, the identity maps on subspaces of $V$ with dimension $r$. In fact, if $\eta, \varepsilon$ are two such idempotents and $\eta \varepsilon=\varepsilon \eta=\varepsilon$, then $\operatorname{ran} \varepsilon=\operatorname{ran}(\varepsilon \eta) \subseteq \operatorname{ran} \eta$. Since $\operatorname{rank} \varepsilon=r=\operatorname{rank} \eta$ and $r$ is finite, it follows that $\operatorname{ran} \varepsilon=\operatorname{ran} \eta$. Therefore, $\varepsilon=\mathrm{id}_{\text {ran } \varepsilon}=\mathrm{id}_{\text {ran } \eta}=\eta$, and this proves that $\eta$ is a primitive idempotent of $I_{r} / I_{r-1}$. Hence, $I_{r} / I_{r-1}$ is completely 0 -simple.

In [2] Vol. 1, pp. 95-96, the authors give a Rees matrix representation for the completely 0 -simple semigroup $T_{r} / T_{r-1}$, where $T_{r}$ is the set of all total transformations $\alpha$ on a finite set $X$ with cardinal $n$ such that rank $\alpha \leq r$ and $2 \leq r \leq n$. And, in effect, [4] Theorem 4.12 provides the Rees matrix representation for the corresponding principal factor in $T(V)$, the semigroup of all total linear transformations of a finite-dimensional vector space $V$. However, that result is expressed in terms of 'independence algebras', an unnecessary complication in the present situation. Therefore, we adopt a simpler approach and proceed to establish a Rees matrix representation for the principal factor $I_{r} / I_{r-1}$ of $I(V)$.

Green's $\mathcal{L}$ and $\mathcal{R}$ relations on $I(X)$ are well-known for any set $X$ : namely, $\alpha \mathcal{L} \beta$ if and only if $\operatorname{ran} \alpha=\operatorname{ran} \beta$; and $\alpha \mathcal{R} \beta$ if and only if $\operatorname{dom} \alpha=\operatorname{dom} \beta$. It is easy to show 
that similar statements hold for $I(V)$ and any vector space $V$ (compare [8] p. 427, for independence algebras). Thus, we have the following result for $I_{r} / I_{r-1}$.

Lemma 6. If $1 \leq r \leq n$ and $\alpha, \beta \in I_{r} / I_{r-1}$ are non zero, then

(a) $\alpha \mathcal{L} \beta$ if and only if $\operatorname{ran} \alpha=\operatorname{ran} \beta$,

(b) $\alpha \mathcal{R} \beta$ if and only if $\operatorname{dom} \alpha=\operatorname{dom} \beta$.

Let $r_{V}$ denote the family of all subspaces of $V$ with dimension $r$, and note that $r_{V}$ is infinite if the field $F$ is infinite, hence our need to consider arbitrary Brandt semigroups. For every $A$ in $r_{V}$, fix a basis $\left\{v_{1}^{A}, \ldots, v_{r}^{A}\right\}$ of $A$. From Lemma $6, I_{r} / I_{r-1}$ has $\left|r_{V}\right|$ nonzero $\mathcal{R}$-classes corresponding to the possible domains of dimension $r$ and $\left|r_{V}\right|$ non-zero $\mathcal{L}$-classes corresponding to the possible ranges of dimension $r$. Let $A, B \in r_{V}$ and put $H_{A, B}=R_{A} \cap L_{B}$. From Lemma $6, \alpha \in H_{A, B}$ if and only if dom $\alpha=A$ and $\operatorname{ran} \alpha=B$, that is, if and only if

$$
\alpha=\left(\begin{array}{ccc}
v_{1}^{A} & \cdots & v_{r}^{A} \\
\sum_{j=1}^{r} x_{1 j} v_{j}^{B} & \cdots & \sum_{j=1}^{r} x_{r j} v_{j}^{B}
\end{array}\right)
$$

for some invertible $r \times r$ matrix $\left[x_{i j}\right]$ over $F$. In this case, we write $\alpha=\left(A,\left[x_{i j}\right], B\right)$.

Now let $\beta \in D_{r}$. Since ran $\alpha \cap \operatorname{dom} \beta$ is a subspace of the $r$-dimensional vector spaces $\operatorname{ran} \alpha$ and $\operatorname{dom} \beta$ (where $r$ is finite and $\beta$ is one-to-one), we have $\operatorname{rank}(\alpha \beta)=r$ if and only if $\operatorname{dim}(\operatorname{ran} \alpha \cap \operatorname{dom} \beta)=r$, and this is true if and only if $B=\operatorname{ran} \alpha=$ $\operatorname{ran} \alpha \cap \operatorname{dom} \beta=\operatorname{dom} \beta$.

Suppose $\operatorname{rank}(\alpha \beta)=r$. Then $B=\operatorname{dom} \beta$ and for some subspace $D$ of $V$ and some invertible $r \times r$ matrix $\left[y_{i j}\right]$ over $F$,

$$
\beta=\left(\begin{array}{ccc}
v_{1}^{B} & \cdots & v_{r}^{B} \\
\sum_{j=1}^{r} y_{1 j} v_{j}^{D} & \cdots & \sum_{j=1}^{r} y_{r j} v_{j}^{D}
\end{array}\right)=\left(B,\left[y_{i j}\right], D\right) .
$$

Also, in this case, $\operatorname{since} \operatorname{ran}(\alpha \beta) \subseteq \operatorname{ran} \beta$ and $r$ is finite, we have $\operatorname{ran}(\alpha \beta)=\operatorname{ran} \beta=D$. Analogously, we conclude that $\operatorname{dom}(\alpha \beta)=\operatorname{dom} \alpha=A$. If $i \in\{1, \ldots, r\}$, then

$$
v_{i}^{A}(\alpha \beta)=\left(\sum_{j=1}^{r} x_{i j} v_{j}^{B}\right) \beta=\sum_{j=1}^{r} x_{i j}\left(\sum_{k=1}^{r} y_{j k} v_{k}^{D}\right)=\sum_{k=1}^{r}\left(\sum_{j=1}^{r} x_{i j} y_{j k}\right) v_{k}^{D} .
$$

Therefore, we have $\alpha \beta=\left(A,\left[z_{i j}\right], D\right)$ where $z_{i j}=\sum_{k=1}^{r} x_{i k} y_{k j}$ and so

$$
\alpha \beta=\left(A,\left[x_{i j}\right]\left[y_{i j}\right], D\right)=\left(A,\left[x_{i j}\right], B\right) \circ\left(\operatorname{dom} \beta,\left[y_{i j}\right], D\right) .
$$

If $\operatorname{rank}(\alpha \beta)<r$, then $B \neq \operatorname{dom} \beta$. Also, $\alpha \beta \notin I_{r} \backslash I_{r-1}$, and hence $\alpha \beta=0$ in $I_{r} / I_{r-1}$. 
Denote the group of all invertible $r \times r$ matrices over $F$ by $G_{r}(F)$, and let $G=G_{r}(F)$ and $J=r_{V}$. Define the mapping $\theta: I_{r} / I_{r-1} \rightarrow B(G, J)$ by $0 \theta=0$ and $\alpha \theta=\left(A,\left[x_{i j}\right], B\right)$ where $v_{i}^{A} \alpha=\sum_{j=1}^{r} x_{i j} v_{j}^{B}$, for every $i \in\{1, \ldots, r\}$. We have shown that $\theta$ is a morphism from $I_{r} / I_{r-1}$ into $B(G, J)$. Clearly, $\theta$ is one-to-one: given $\alpha, \beta \in I_{r} \backslash I_{r-1}$ such that $\left(\operatorname{dom} \alpha,\left[x_{i j}\right], \operatorname{ran} \alpha\right)=\alpha \theta=\beta \theta=\left(\operatorname{dom} \beta,\left[y_{i j}\right], \operatorname{ran} \beta\right)$, we have

$$
v_{i}^{\operatorname{dom} \alpha} \alpha=\sum_{j=1}^{r} x_{i j} v_{j}^{\mathrm{ran} \alpha}=\sum_{j=1}^{r} y_{i j} v_{j}^{\mathrm{ran} \beta}=v_{i}^{\operatorname{dom} \beta} \beta .
$$

Since $\operatorname{dom} \alpha=\operatorname{dom} \beta$, it follows that $\alpha=\beta$. To see that $\theta$ is onto, let $\left[x_{i j}\right]$ be an invertible $r \times r$ matrix over $F$ and $A, B \in r_{V}$. Now define $\alpha \in P(V)$ by

$$
\alpha=\left(\begin{array}{ccc}
v_{1}^{A} & \ldots & v_{r}^{A} \\
\sum_{j=1}^{r} x_{1 j} v_{j}^{B} & \ldots & \sum_{j=1}^{r} x_{r j} v_{j}^{B}
\end{array}\right) .
$$

Then, dom $\alpha=\left\langle v_{1}^{A}, \ldots, v_{r}^{A}\right\rangle=A$ and, since $\left[x_{i j}\right]$ is invertible, $\alpha$ is one-to-one and ran $\alpha=\left\langle v_{1}^{B}, \ldots, v_{r}^{B}\right\rangle=B$. Therefore, $\alpha \in I_{r} \backslash I_{r-1}$ and $\alpha \theta=\left(A,\left[x_{i j}\right], B\right)$. Hence $\theta$ is an isomorphism from $I_{r} / I_{r-1}$ onto $B(G, J)$.

Finally, we turn to the maximal inverse subsemigroups $S$ of $I_{r}$ where $r<n$ (the case $r=n$ is handled by Theorem 3). If $I_{r-1} \backslash S \neq \emptyset$ then $S \varsubsetneqq S \cup I_{r-1}$, which is an inverse subsemigroup of $I_{r}$. By the maximality of $S$, this implies $S \cup I_{r-1}=I_{r}$. Hence $D_{r} \subseteq S$ and, by Lemma 1, this implies $I_{r} \subseteq S$ (see the remark after the proof of Theorem 4 above). Since this contradicts our supposition, we deduce that $I_{r-1} \subseteq S$ and thus $S=I_{r-1} \cup\left(S \cap D_{r}\right)$. Clearly, $\left(S \cap D_{r}\right) \cup\{0\}$ is an inverse subsemigroup of $I_{r} / I_{r-1}$, and we assert it is a maximal one. For, if $T$ is an inverse subsemigroup of $I_{r} / I_{r-1}$ which properly contains it, then $I_{r-1} \cup(T \backslash\{0\})$ is an inverse subsemigroup of $I_{r}$ (under composition) which properly contains $S$, a contradiction.

If $\operatorname{dim} V=n$ and $r=n$ then $I_{r} / I_{r-1}$ is simply the group $G_{n}$ of units in $I(V)$ with 0 adjoined. Therefore, $|J|=1$ in the representation of $G_{n}^{0}$ as a Brandt semigroup $B(G, J)$, and so Theorem 4 is not applicable in this case. However, that result describes all maximal inverse subsemigroups of the Brandt semigroup $B(G, J)$ where $G$ is an arbitrary group and $|J| \geq 2$; hence, via the isomorphism established above, we know all maximal inverse subsemigroups of $I_{r} / I_{r-1}$ where $r<n$. Consequently, we have the following result.

Theorem 5. Let $1 \leq r<n=\operatorname{dim} V$ and let $r_{V}$ denote the family of all subspaces of $V$ with dimension $r$. If $S$ is a subsemigroup of the inverse semigroup $I_{r}$, then $S$ is a maximal inverse subsemigroup of $I_{r}$ if and only if $S$ is one of the following: 
(a) $S=I_{r-1} \cup B_{r_{V_{1}}} \cup B_{r_{V_{2}}}$, where $\left(r_{V_{1}}, r_{V_{2}}\right)$ is a two-partition of $r_{V}$ and $B_{r_{V_{i}}}=\left\{\alpha \in I_{r}\right.$ : $\operatorname{dom} \alpha$, ran $\left.\alpha \in r_{V_{i}}\right\}$ for $i=1,2$,

(b) $S=I_{r-1} \cup\left\langle\left\langle H_{A A}^{*} \cup\left\{\alpha_{B}: B \in r_{V}^{\prime}\right\}\right\rangle\right\rangle$, where $A \in r_{V}, r_{V}^{\prime}=r_{V} \backslash\{A\}, H_{A A}^{*}=\{\alpha=$ $\left[h_{i j}\right] \in I_{r}: \operatorname{dom} \alpha=A=\operatorname{ran} \alpha$ and $\left.\left[h_{i j}\right] \in H\right\}, H$ is a maximal subgroup of the group $G_{r}(F)$ of all $r \times r$ invertible matrices over $F$ and $\left[\alpha_{B}\right] \in I_{r}^{r_{V}^{\prime}}$ where dom $\alpha_{B}=A$ and $\operatorname{ran} \alpha_{B}=B$ for every $B \in r_{V}^{\prime}$.

\section{References}

1. Z. I. Borevich, Some subgroups of the full linear group. (Russian), Zap. Naučn. Sem. Leningrad Otdel. Mat. Inst. Steklov (Notes of Scientific Seminars, Steklov Math. Inst., USSR Acad. Sc., Leningrad), 71 (1977), 42-46; English translation: J. Sov. Maths, 20 (1982), 2528-2532.

2. A. H. Clifford and G. B. Preston, The Algebraic Theory of Semigroups, Mathematical Surveys, No. 7, Vols. 1 and 2, American Mathematical Society, Providence, RI, 1961 and 1967.

3. G. M. S. Gomes and J. M. Howie, On the ranks of certain finite semigroups of transformations, Math. Proc. Cambridge Philos. Soc., 101 (3)(1987), 395-403.

4. V. Gould, Independence algebras, Algebra Universalis, 33 (3)(1995), 294-318.

5. T. W. Hungerford, Algebra, Springer-Verlag, NY, 1974.

6. P. Kleidman and M. Liebeck, The subgroup structure of the finite classical groups, London Mathematical Society Lecture Note Series Vol. 129, Cambridge University Press, Cambridge, 1990.

7. G. Köthe, Topological vector spaces, 2 Vols., Springer Verlag, Heidelberg, 1969.

8. L. M. Lima, Nilpotent local automorphisms of an independence algebra, Proc. Royal Soc. Edinburgh, 124A (3)(1994), 423-436.

9. M. P. O. Marques-Smith and R. P. Sullivan, The ideal structure of nilpotent-generated transformation semigroups, Bull. Austral. Math. Soc., 60 (2)(1999), 303-318.

10. X. Yang, A classification of maximal inverse subsemigroups of the finite symmetric inverse semigroups, Comm. Algebra, 27 (8)(1999), 4089-4096. 
11. A. E. Zalesskiı̌, Linear groups, pp. 97-196 in Algebra IV, ed A. I. Kostrikin and I. R. Shafarevich, Encyclopedia of Mathematics and Science, Springer-Verlag, Berlin, 1993. 